\title{
Algorithm to Increase Energy Efficiency and Coverage for Wireless Sensor Network
}

\author{
Aphrin S Pathan ${ }^{1}$, Shabda Dongavkar ${ }^{2}$ \\ ${ }^{1}$ Savitri bai phule University Pune, Student at G.H.Raisoni college Of Engineering and Managemet, Wagholi, Pune \\ ${ }^{2}$ Professor, Savitri bai Phule University Professor at G.H Raisoni College of Engineering and Management, Wagholi, Pune
}

\begin{abstract}
A wireless sensor network (WSN) (sometimes called a wireless sensor and actor network (WSAN)) are distributed autonomous sensors to monitor physical or environmental conditions, such as temperature, sound, pressure, etc. and to cooperatively pass their data through the network to a main location. Wireless network can perform multipath routing in short distance to save energy and a power of particular node. The reason behind this Is network's nodes contain less battery power and energy. Sensor nodes are very small and fitted in large network area which can be static or can move so it is not easily possible to charge them frequently. For better performance of a network by saving the communication and processing power is only to increase node's life time. Power or energy consumptions are main issues for routing data packet in the network which we need to consider. Mainly WSN works on a large scale network area in which sensor nodes are deployed geographically. Reason Behind to develop the separate protocols for WSN is that user cannot deploy directly MANET routing protocol in WSN. For saving the power of sensor nodes many technologies were developed. Study of these technologies, algorithms and methods are introduced in this paper.
\end{abstract}

Keywords: Mobile AD-hoc Network, WSN, Routing Protocol, Power Aware Routing

\section{Introduction}

The main reason of development from wired LANs to wireless LANs (WLANs) is due to moving technology. In Other words person can work from office as well from outside the office, which has provided flexibility in working scenario. Wired LANs are those which provide high bit rate to fulfill requirements of bandwidth consuming application like video conferences, streaming online video, downloading high bit rate movies, pictures etc. within a second.

Ad-hoc Sensor Network or wireless Sensor Network is the combination of hundreds and thousands of small sensors [1]. These small (tiny) sensor nodes have ability to either communicate with one to another sensor or direct communication with the base station (BS) connected externally. Sensor nodes have small size . They are having poor battery power, used to communicate in small area of short distance. According to their functionality sensors can be of same type or different. Sensors are the nodes which perform some kinds of task such as sensing of specific type of data, data processing, and communicating components. A sensor network is made with the combination of many sensors that are densely used to deploy inside the geographic environment, this area in which sensors are deployed and connected with each other called sensor field.

\section{A. Application of Sensor Network [2] :}

- They are used in military applications for guiding the system of intelligent missiles, and finding the attacks by weapons, such as nuclear, biological or chemical.

- In forest for controlling fire and air and water pollution environmental monitoring application Sensors are used.

- Sensors can be used in patient body for diagnosis and monitoring.

\section{B. Classification of Routing Protocols in WSN:-}

There are mainly three basic types of Routing Protocols [3], [4], [5] defined in wireless sensor network:-

a) Flat based

b) Hieratical based

c) Location based

\section{Introduction about Protocols}

\section{A. Flat based}

Flat routing protocol is a network communication protocol implemented by routers in which all routers are each other's peers.

Flat routing protocol distributes routing information to routers that are connected to each other without any organization or segmentation structure between them. Flat based routing protocols are given following-

1. Flooding \& Gossiping: - In flooding mechanism source node send a broadcast message which consist maximum hop count number to destination node. This message received by each node between source to destination and each node broadcast this message if maximum hop count is not reached and also that node is not destination node. Flooding does not maintain any complex topology and route discovery algorithm in the network.

Flooding has some disadvantages given following-

- Implosion:-Implosion i.e. duplicate message arrive to the same node.

- Overlap:-overlapping i.e. Same event sensed by more than one node.

- Resource blindness:-Because of redundant transmission of data packets it reduces the network lifetime. 


\section{International Journal of Science and Research (IJSR) \\ ISSN (Online): 2319-7064}

Index Copernicus Value (2013): 6.14 | Impact Factor (2014): 5.611

Gossiping : is an enhanced version of flooding in which nodes do not broadcast message to all node but send a packet to selected node only it solves the problem of implosion but time taken to propagate message throughout network is large. Gossiping consist lower overhead then flooding.

2. SPIN:-It is called as Sensor Protocol for Information via Negotiation. SPIN protocol is introduced to reduce the flooding protocol problem like overlap and implosion via negotiation, this protocol also prolong the network lifetime by introducing threshold based resource aware operation.

3. Direct Diffusion: In direct diffusion protocol nodes perform individual operation instead of all operations performed by base station. In this protocol Request and queries for data sensed by node generated by sensor node itself instead of all request and queries generated by base station. In direct diffusion technique interest gradient parameter is used. Data comes on each sensor node is named by one or more attributes value and if other node is interested in this attribute then that node will express their interest. This attribute is nothing but a set of description for the data.

\section{Energy Aware Routing:}

Best way to minimize the energy cost for communication and for increasing the network lifetime, energy aware routing protocol is introduced. In energy aware routing protocol data transmission is done by selecting multiple numbers of optimal paths instead of transmitting from one optimal path. Probability function decides the path for data transmission.

\section{Energy efficient Geocast Routing:}

This protocol is based on operation in which packets are delivered to a group of nodes situated in geographical area within a specified limit.I-minrouting protocol comes under this routing category.

\section{B. Hierarchical based Routing (Clustering):-}

Hierarchical routing is the procedure of arranging routers in a hierarchical manner. A good example would be to consider a corporate intranet. Most corporate intranets consist of a high speed backbone network. Connected to this backbone are routers which are in turn connected to a particular workgroup. These workgroups occupy a unique LAN. The reason this is a good arrangement is because even though there might be dozens of different workgroups, the span (maximum hop count to get from one host to any other host on the network) is 2 .

Even if the workgroups divided their LAN network into smaller partitions, the span could only increase to 4 in this particular example. Given following protocols comes in this category-

\section{LEACH \& TL-LEACH:}

LEACH protocol is also called Low-Energy Adaptive clustering Hierarchy. This is the first developed protocol based on clustering method. Protocol works on probability function each node has a probability to select cluster head. Because every node has equal probability to select cluster head in each round cluster head change. TL-LEACH is advanced protocol of LEACH known as Two Level LEACH protocol in which cluster head is divided into two part.

- first one is known as primary cluster head

- Second one is known as secondary cluster head.

It reduces the number of nodes that transmits the data to the base station so energy dissipation is low as compare to LEACH.

Disadvantage of LEACH over TLLEACH: it takes more energy for formation of cluster head. TLLEACH reduces the energy dissipation in some limit as compare to LEACH.

\section{PEGASIS \& H-PAGASIS:-}

PEGASIS stand for Power Efficient Gathering in Sensor Information System. This protocol is an improvement on LEACH protocol based on optimal chain based protocol. LEACH protocol works on clustering technology but PEGASISconstruct a chain of nodes in which each nodes communicate only with his neighbour node close to it. So it reduces the amount of energy used per round. For constructing chain of nodes greedy method is used. PEGASIS works much better as compare with LEACH but time taken for transmission of data is higher which prolonged the network lifetime.

H-PEGASIS (Hierarchical PEGASIS) is an improvement on PEGASIS which allow concurrent transmission of data when nodes are not adjacent.

\section{TEEN\& APTEEN:-}

TEEN protocol is known as Threshold Sensitive Energy Efficient Sensor Network Protocol first developed for reactive networks. This protocol used for temperature sensing application. This protocol is developed to increase the energy efficiency in wireless sensor network. TEEN is developed for reactive network. Transmission in TEEN mainly depends on two parameters are hard threshold value and soft threshold value. APTEEN (Adaptive Threshold Sensitive Energy Efficient Sensor Network Protocol) is an extension of TEEN. In APTEEN when cluster head is decided it broadcast all the parameters such as threshold values, count time and attributes to all over the nodes. The working performance of APTEEN lies between LEACH and TEEN protocol.

Both protocol (LEACH and TEEN) have one disadvantage that algorithm contains overhead and complexity when forming cluster.

4. HEED: - HEED protocol works on remaining energy of each sensor node in the network. Protocol works on energy efficiently in the network. Cluster head selection is based on parameter of remaining energy of sensor node. HEED protocol implemented on tiny-OS. Node degree or average distance is used as a parameter. Two sensor nodes have same remaining energy in the network after one transmission has been done. In the HEED protocol it is calculated that clustering and data aggregation at least double the lifetime in the wireless sensor network. In HEED if Cluster node is near to the base station then node directly communicate with the base station and discharge quickly and energy is wasted that is used for formation cluster head. 5. HRDCS: - A hybrid 


\section{International Journal of Science and Research (IJSR) \\ ISSN (Online): 2319-7064}

Index Copernicus Value (2013): 6.14 | Impact Factor (2014): 5.611

relative distance based cluster scheme in wireless sensor networks which save the energy efficiently. Energy efficiency has great importance in this protocol. In HRDCS node construct the cluster for data collection and transmission in the network. Cluster head selected on the basis of transmission range from the base station. HRDCS works on distance between $\mathrm{CH}$ and $\mathrm{BS}$ and energy used at the time of cluster head construction.

\section{c. Location based Routing:}

For static i.e. constant network most of the time there is no need to care about exact location information of any node but in mobile i.e. moving network like wireless sensor network in which sensor nodes are movable then its compulsory to keep the information about their locations. Location information contains distance between two different nodes for routing data. Distance is calculated on the basis of signal strength so that energy consumption is calculated.

Following types of protocol comes in this category-

1. MECN \& SMECN:-A location based routing protocol MECN (Minimum Energy Communication Network) as name implies works by using minimum energy for wireless sensor network and by utilizing low power GPS. This protocol is designed for mobile network by keeping the location information but it is best work for sensor network because sensor nodes are not mobile. MECN transmit data through relay region which consist nodes in a surrounding area. In place of direct transmission, transmission through relay region node saves the energy of node. MECN is self reconfiguration protocol which dynamically solves the nodes failure and self adjust the deployment of new sensors.

2. GEAR:-Geographical Energy Aware Routing is an improvement on direct diffusion method by adding geographical data of node. GEAR helps data packet to forward towards the targeted nodes only this technique helps for balancing the energy consumption for forward data packets and increase the network lifetime.

3. GAF \& H-GAF: - GAF protocol is defined as geographical adaptive fidelity protocol. This protocol works on this method in which from large number of sensor nodes only few nodes are selected for transmission of data and other nodes are sleep so the network size become small when transmit data and saves nodes battery when nodes not participating in communication.

H-GAF (hierarchical geographical adaptive fidelity) is an improved version of GAF which saves much more energy as compare to GAF. HGAF saves the battery power and node energy by enlarging the cell size and layered structure of GAF.

\section{Power aware routing metrics [6]:-}

To save energy of sensor node for making network lifetime greater some metrics or parameters are considered to being improved by algorithms.

These parameters are as given following:-

- Minimum energy consumption per packet of any node should be there.

- Maximizing Network connectivity intended for each lump.
- Minimum variance in node authority levels should be there.

- Cost per packet should be minimum.

- Communication delay should be minimum

\section{Related Work}

Patel Dhavalet. al. [7] has proposed an algorithm to improve the performance of routing protocol based on demand. AODV protocol was protocol used to implement this approach in place of DSR protocol because AODV removes unnecessary information quickly and perform better with extra load. This approach mainly based on, to find neighboring nodes with proper routing path to the destination. Source node finds the energy level of other nodes by broadcasting the message. A threshold value by default defined used for comparison with energy level of other nodes, if any node contains energy level higher then threshold value is selected with less distance. It uses both distance and energy metric.

S. Yessadet. al. [8] and Samira Yessadet. al. [9] has proposed an EAR-Energy Aware Routing protocol is a reactive protocol, initiated by destination. Main idea behind EAR is to choose optimal path between available several paths found, with the probability of being chosen as energy metric assigned to the each path. So no duplicate node get selected next time. FEAR-Fair Energy Ware Routing is an advanced version of EAR, which used to improve the network lifetime. It reduces the probability used in the high demanded source node. FEAR reduces the probability of use of many nodes belong to several routes in the network. BEER is other routing protocol stands for Balanced Energy Efficient Routing protocol. It differ from FEAR protocol just from one parameter that is N sent in NFTM (number forwarding table message) message.

Shah AhsanulHaqueet. al. [10] has proposed a method in which large numbers of heterogeneous nodes are consider for communication via radio signals. Each cluster formed by sensor node has one cluster head which is the storage node. Storage node is capable for storing and processing data and information. Sensor nodes select their cluster head and cluster according to the energy level and distance. This method work with wireless environment and does not consider the mobility of nodes.

Method includes four steps:-

- Network model

- Cluster Head Selection

- Cluster Construction

- Sensing Scenario

- Data transfer

I Sakthideviet. al. [11] has proposed a Fuzzy Based Trust Aware Routing Framework (FBTARF) for improvement in security of dynamic WSN .FBTARF also provides energy efficient routing and reliable trust provided by using fuzzification methods. Fuzzy based methods consider multiple constraints which provide better security and energy conservation. It also provides effective and efficient routing in dynamic WSN. 


\section{International Journal of Science and Research (IJSR) \\ ISSN (Online): 2319-7064 \\ Index Copernicus Value (2013): 6.14 | Impact Factor (2014): 5.611}

Nikhil Marriwala et. al. [12] has introduced a modified LEACH protocol which performs some modification in simple LEACH protocol. Simple LEACH protocol is based on clustering method in which clusters-Head are formed randomly. LEACH protocol divided WSN data into several clusters, each cluster select a cluster-head which aggregate data coming from the cluster nodes, process it and send it to the base station. LEACH uses a random selection process in place of fix method. Modified LEACH works on fix manner which increase the WSN lifetime. In modified LEACH protocol cluster head is choose on the bases of its maximum residual energy and minimum distance from the sensor nodes.

W. Chee-Wah Tan et. al. [13] has proposed a heuristic scheme Power and Mobility Aware Routing or PMAR protocol. This is able to control packet flooding during route discovery and link breakage because of node mobility. PMAR works like an optimization in static network PMAR able to reduce the network overheads.

Yang wenguoet. al. [14] has introduced a tuning transmission radii solution scheme for the purpose of energy conservation for solution, a semi grid network is constructed as an application and routing algorithm designed which tries to calculate the uniform of nodes energy consumption and then result is compared.

Tseng-Yi Chen et. al. [15] has proposed two energy efficient geographic routing algorithm (EEGRA) for WSN. It is based on geographic routing. Geographic routing algorithms have a low computation and storage requirement which fits for WSN environments. To measure the power consumption SINR (Signal to Interference and Noise Routing) was used.

Xia Li et. al. [16] has introduced an AECRP (Adaptive Energy Efficient Clustering Routing Protocol). Basic concept of AECRP is based on LEACH but AECRP works on particle swarm clustering method which uses improved particle swarm optimization (PSO) method.

Clustering works by using two approaches-

1. Choosing cluster heads.

2. Self organization clustering.

Choosing the clustering head is a core part of clustering algorithm. This is based on improved PSO algorithm. Evaluation function is designed whose independent variable calculated by remaining energy of nodes, the distribution within a cluster and among clusters. The particle whose evaluation function is large will be better for selection. So node whose energy is high or sufficient and position is appropriate can be selected as a cluster head.

Y. Khan et. al. [17] has proposed Location Aware Permanent $\mathrm{CH}(\mathrm{LPCH})$ and User Defined Location Aware Permanent $\mathrm{CH}$ (UDLPCH). Both protocols work on clustering method. Physically network field is divided into two reasons in LPCH. Nodes are selected equally randomly in each reason for deploying in network. Initially protocol works on LEACH protocol for first round $\mathrm{CH}$ are selected according to LEACH algorithm. Numbers of $\mathrm{CH}$ are constant from first to last round. This increases the network stability and also gives greater throughput. UDLPCH is same as LPCH but differ in that, From starting or initial round user can define optimal number of CH's in each reason. In case of UDLPCH greater stability period and throughput obtained as compare to LPCH.

MdNafees Rahman et. al. [18] has proposed a concept of Relay Node in conjunction with PSO based algorithm. In multi-hop network communication the data collected from different sensor nodes in the network reach to the sink node through the nodes those are near from the sink node, because these nodes send and receive data frequently so it's greater possibility that they will die soon, because they pass a huge amount of data. In this paper a simple concept of relay node proposed which improved the network lifetime. Relay node collect data from different sensor nodes and send it to the Sink node, so it reduces the data traffic of sensor nodes especially for those nodes which are near to the sink. Hence energy consumption of sensor nodes decreases and lifetime increases of the network. To find the optimal location from relay node to sink node is done by using PSO- particle swarm optimization algorithm.

ShobhaPoojaryet. al. [19] has introduced the scheme of routing protocol for wireless multimedia sensor network (WMSN) the proposed algorithm called Multipath Data Transfer (MPDT). MPDT introduced technique to select multiple path from source to destination to transmit data in selected multiple paths. Protocol immune from some specific attacks also. Protocol works on basically in two parts -1 . Route set-up phase: - In which if multiple paths available then a path is set using maximum residual energy. 2. Data Transmission phase: - To transfer data from source to destination, data is split into $\mathrm{m}$ parts if $\mathrm{m}$ nodes are available in selected multi-path. To secure data RS (Reed Solomon) Encoding is used.

H. Y. Bohariet. al. [20] has proposed an algorithm in which distance is calculated from each of its neighbours from source to destination and also calculate minimum energy required to send the data packet which contained appropriate additional information. Higher energy node is selected with minimum distance compared by threshold value for routing data packet from selected path. Distance between two nodes in earth surface is calculated by its longitude and latitude coordinates.

\section{Conclusion and Future Work}

Many researchers introduced routing techniques as discussed above for improvement in different parameters related to network node for example Energy, Delay, Throughput, Bandwidths, Transmission time. Routing with energy awareness is latest topic in the research to increase the network lifetime and performance. Sensor network can not adopt only single protocol globally so need to design a protocol which satisfies all conditions and parameters. In future we are going to improve Routing Algorithm with considering power aware metrics for example-delay, transmission time, link cost and energy aware parameters to increase the node's lifetime, reliability of data, increase the throughput, higher packet delivery ratio. 


\section{International Journal of Science and Research (IJSR) \\ ISSN (Online): 2319-7064}

Index Copernicus Value (2013): 6.14 | Impact Factor (2014): 5.611

\section{References}

[1] Ian F. Akyildiz, Weilian Su, Yogesh Sankarasubramaniam, and ErdalCayirci -“A survey on sensor network" IEEE Communications Magazine August 2002.

[2] C.Siva Ram Murthy and B.S. Manoj, Ad hoc Wireless Network, 2nd, Ed. Pearson, Twenty First Impressions, 2013.

[3] Rajashree.V.Biradar , V.C .Patil , Dr. S. R. Sawant , Dr. R. R. Mudholkar ," Classification And Comparison Of Routing Protocols In Wireless Sensor Networks," Special Issue on Ubiquitous Computing Security Systems.

[4] JyotiSaraswat, Neha Rathi\&ParthaPratim Bhattacharya," Techniques to Enhance Lifetime of Wireless Sensor Networks: A Survey," Global Journal of Computer Science and Technology Network, Web \& Security Volume 12 Issue 14 Version 1.0 Year 2012.

[5] Sitender Kumar, Arvind Kumar, Rohitkumarvishwkarma," A Survey on Routing Protocol for Wireless Sensor Network," International Journal of Advanced Research in Computer Engineering \& Technology (IJARCET) Volume 2, Issue 2, February 2013.

[6] Karamvir Kaur, Inderdeep Kaur Aulakh, "Power Aware Metrics and Routing Techniques in MANETs", IEEE 2011.

[7] Patel Dhaval , Rana Arpit ,'Power Aware Routing Protocol to Extend Life-Time of MANET," International Journal of Engineering Research \& Technology (IJERT) Vol. 1 Issue 10, ISSN: 2278-0181, December- 2012.

[8] S. Yessad, L. Bouallouche-Medjkoune, and D. Assani, "proposition and evaluation of a novel routing protocol for wireless sensor networks,'International Workshop on Verification and Evaluation of Computer and Communication Systems VECoS'11, Tunis, Tunisie, Sep 15-17, 2011.

[9] Samira Yessad, NassimaTazarart, LyesBakli, LouizaMedjkouneBouallouche and DjamilAissani," Balanced Energy Efficient Routing Protocol for WSN," the 2nd International Conference on Communication and Information Technology (ICCIT), IEEE 2012.

[10] Shah AhsanulHaque, SyedMahfuzul Aziz," Storage Node Based Routing protocol for Wireless Sensor Network," Seventh International Conference on Sensing Technology, IEEE 2013.

[11]I.Sakthidevi, E.Srievidhyajanani,"Secured Fuzzy Based Routing Framework for Dynamic Wireless Sensor Networks," International Conference on Circuits, Power and Computing Technologies [ICCPCT-2013] ,IEEE 2013.

[12] NikhilMarriwala, Priyanka Rathee,"An Approach to Increase the Wireless Sensor Network Lifetime," World Congress on Information and Communication Technologies, IEEE 2012.

[13] W. Chee-WahTan , S.K. Bose ,T.-H. Cheng," Power and mobility aware routing in wireless ad hoc Networks," Published in IET (The Institution of Engineering and Technology 2012) Communications Received on 20th June 2011 Revised on 17th February 2012 .

[14] Yang wenguo, GuoTiande," The Non-uniform Property of Energy Consumption and its Solution to the Wireless
Sensor Network,"Second International Workshop on Education Technology and Computer Science, IEEE 2010.

[15] Tseng-Yi Chen, Hsin-Wen Wei, Che-Rung Lee, Fu-Nan Huang, Tsan-sheng Hsu, Wei-Kuan Shih," EEGRA: Energy Efficient Geographic Routing Algorithms for Wireless Sensor Network," International Symposium on Pervasive Systems, Algorithms and Networks, IEEE 2012.

[16]Xia Li, Wang Gang, Liu Zongqi, Zhang Yanyan,” An energy efficient routing protocol based on particle swarm clustering algorithm and inter-cluster routing algorithm for WSN," 25th Chinese Control and Decision Conference (CCDC) IEEE 2013.

[17] Y. Khan, N. Javaid, M. J. Khan_, Y. Ahmad, M. H. Zubair, S. A. Shah," LPCH and UDLPCH: Locationaware Routing Techniques in WSNs," Eighth International Conference on Broadband, Wireless Computing, Communication and Applications, IEEE 2013.

[18] MdNafees Rahman, M A Matin ," Efficient Algorithm for Prolonging Network Lifetime of Wireless Sensor Networks," TSINGHUA SCIENCE AND TECHNOLOGY ISSN11100702141101/1211Volume 16, Number 6, December 2011.

[19] ShobhaPoojary, ManoharaPai M M,"Multipath Data Transfer in Wireless Multimedia Sensor Network,", 2010 International Conference on Broadband, Wireless Computing, Communication and Applications, IEEE 2010.

[20]H. Y. Bohari, Sh. O. S. Khanna ," Energy Efficient Power Aware Routing Algorithm (EEPARA) For Mobile Ad-Hoc Network (MANET) , " International Journal of Engineering Research \& Technology (IJERT) Vol. 2 Issue 7, July - 2013 